\title{
High dose rate (HDR) brachytherapy in gynecologic cancer regression: a review of the literature
}

\author{
Mari Uyeda ${ }^{1,2^{*}}$ D, Fabian Friedrich ${ }^{3,4}$ and Antonio Cassio Assis Pellizzon ${ }^{1,2}$
}

\begin{abstract}
Brachytherapy as a form of treatment for gynecological tumors has been used for a long time in Brazil (since 1991) and can be considered as a form of radiotherapy treatment. High Dose Rate (HDR) brachytherapy aims at delivering high dose rates of radiation in restricted volumes of the body, thereby increasing disease control and lower treatment toxicity for adjacent normal tissues. Cervical cancer (CC) is a disease that still affects women in developing countries and, despite being detected by laboratory and imaging tests, in many developing countries these techniques are not yet accessible to all that are affected by cervical cancer. HDR presents important results when isolated or in association with other treatment techniques. Numerous studies have shown that HDR for gynecological cancer presents results that can reach up to $85 \%$ cure, and with this it is concluded that there are few complications during or after treatment, since adjacent tissues are preserved, making HDR a safe procedure for patients and professionals.
\end{abstract}

Keywords: Brachytherapy, Gynecological cancer, Radiotherapy, Cervical cancer, Endometrial cancer

\section{Background}

High Dose Rate brachytherapy (HDR) is a therapeutic modality of radiotherapy in which radioactive sources are used in close contact with the region to be treated. The aim of this treatment is to administer high doses of radiation in restricted volumes of the organism, to have greater control of the disease and less toxicity of the treatment to the adjacent normal tissues [1].

Over time, brachytherapy has been consolidated and has become a safe technique for patients with cervical tumors and with very satisfactory results of regression and even cure of the tumor [1].

\section{Cervical Cancer}

Cervical cancer $(\mathrm{CC})$ is unique among malignant disease because risk factors are very well established, it has a prolonged preinvasive state that can be detected through screening, the etiology of the disease is known and prophylactic vaccines are available $[2,3]$. The symptoms

\footnotetext{
* Correspondence: mariuyedanutri@gmail.com

${ }^{1}$ AC Camargo Cancer Center, Tamandaré St, 753 - Liberdade, São Paulo 01509-020, Brazil

${ }^{2}$ Department of Radiotherapy of the Hospital AC Camargo Cancer Center,

São Paulo, Brazil

Full list of author information is available at the end of the article
}

reported by women with locally advanced disease (FIGO IB2-IVA) include flank pain, lower extremity swelling, hematuria, and/or rectal bleeding. Cisplatin-based chemoradiation plus high dose rate intracavitary brachytherapy can prevent locoregional relapse and effect cure in $40-75 \%$ of patients $[4,5]$.

Surgery as sole treatment is indicated for the initial stages (carcinoma in situ, micro-invasive, and invasive stage IB1), but depending on the diameter of the lesion, some centers treat IIA1 cancers surgically [6]. However, this approach is not recommended as initial therapy of IB2 tumors (limited to the cervix and having a diameter above $4 \mathrm{~cm}$ ). For lesions in stage 0 (in situ carcinoma), conization with free margins is sufficient $[7,8]$.

In stage IA1 the choice will depend on the patient's desire to preserve fertility, and whether there is lymphovascular invasion. After conization, if the cone margins are free and there is no lymphovascular invasion, clinical monitoring alone is recommended. If there is no interest in preserving fertility, the recommendation is that the surgery should include plain hysterectomy. Bilateral salpingectomy associated with hysterectomy to prevent ovarian carcinoma is also recommended [9]. However, if there is lymphovascular invasion, radical hysterectomy 
and pelvic lymphadenectomy, or sentinel lymph node technique can be indicated. If the patient wants to preserve fertility, radical trachelectomy can be offered [9].

As for IA2 and IB1 stages, for patients who do not want to preserve fertility, the best alternative is radical hysterectomy class C, by Querleau and Morrow [10] with resection of parametrium at the level of internal iliac artery, which corresponds to the classical Werteim-Meigs operation, or type III-V Piver-Rutledge, in addition to pelvic lymphadenectomy [9]. For these stages (IA2 and IB1), the sentinel lymph node technique can be proposed to prevent radical lymphadenectomy and risks of associated morbidities (evidence and recommendation 2B for sentinel lymph node). In these stages, if the patient has a clinical contraindication or if she does not accept the surgery, the choice becomes exclusive radiotherapy, using teletherapy supplemented by brachytherapy. Radical surgical procedures can be performed by laparotomy or laparoscopy, including robotic surgery [11].

Surgery as initial treatment is not indicated for stages IB2, IIA1 and IIA2 cancers. The probability of positive margins or other indications for radiotherapy or chemotherapy in these stages is very high, around $80 \%$. The addition of adjuvant therapies to surgery (chemoradiation) increases morbidity, worsening the quality of life of the patient [12].

An important component of the treatment of cervical carcinoma in the early stages is adding radiation therapy in situations at high risk for local or systemic recurrence. Poor prognostic indicators are obtained from surgical specimens and include the following: positive pelvic lymph nodes, parametrial involvement or positive surgical margin. More recently, another category of prognostic indicators was added to clinical practice and applies to patients without any of the cited criteria. These, considered as minor criteria, are: diameter of the primary tumor associated with lymphovascular invasion, or deep invasion of the cervical stroma [6]. The presence of any of the major criteria, or a combination of the Sedlis criteria, is an indication for adjuvant pelvic radiotherapy plus teletherapy associated with brachytherapy in cases of worse prognosis. The addition of concurrent chemotherapy with radiation therapy (cisplatin in weekly doses) for cases with higher risk of recurrence, especially for patients with more than one positive lymph node, showed benefits in terms of overall survival and recurrence-free interval [6].

\section{Brachytherapy}

Brachytherapy is a key component of radiotherapy treatment for cervical cancer stages IB and greater [13]. This category of disease represents $60 \%$ of cervical cancer cases in developing countries - twice as many as the $30 \%$ seen in developed nations [14]. Thus, having access to high quality brachytherapy is essential for effective disease management. Given the widespread unmet surgical need, disproportionately in LMIC, the therapeutic potential of brachytherapy is of particular significance [14]. The comparison of EBRT followed by radical hysterectomy or standard brachytherapy in stage IB2 to IIB CC patients, failed to show superiority of radical hysterectomy over brachytherapy related to survival free progression and overall survival [15]. In the case of LMIC, CC alone counts for up to $7 \%$ of the patients with indications for radiotherapy [16]. Small et al. recently commented on the lack of radiotherapy equipment to meet the cervical burden in LMIC and a high-dose rate (HDR) brachytherapy unit capable of treating roughly 10-12 cases per day [9].

The advantage of brachytherapy comes from its dosimetric benefits, including the ability to deliver a locally high and conformal dose to the site of disease with a rapid dose fall-off, thereby sparing adjacent structures such as the bladder, rectum, sigmoid, and small bowel. Brachytherapy remains unavailable in many countries. Even in countries in which brachytherapy is easily accessible, its use is declining.

\section{HDR brachytherapy versus LDR brachytherapy}

When starting the brachytherapy component of treatment, one must first decide on whether to use HDR or LDR brachytherapy. Historically, cervical brachytherapy used exclusively LDR sources. Treatments were delivered over 1-2 fractions, with treatment times of (typically) 1-3 days, requiring prolonged patient immobilization and hospitalization [17]. LDR brachytherapy is delivered at a point A dose rate of $<0.4 \mathrm{~Gy} /$ hour, typically using the cesium-137 isotope [17].

Since the early 2000s, there has been increasing adoption and utilization of HDR, as opposed to LDR. Eighty-five percent of respondents to a recent American Brachytherapy Society (ABS) survey reported having HDR at their institution [17].

HDR brachytherapy, a remote afterloading technology, allows a small iridium source attached to the end of a cable to be robotically driven through multiple channels, stopping at predetermined points (dwell positions) for varied lengths of time [17].

HDR brachytherapy is delivered at a point A dose rate of $>12$ Gy/hour, primarily using the iridium-192 isotope. The advantages of HDR brachytherapy include the precise positioning of the source, infinitely variable dwell times and dwell positions - allowing for "dose sculpting" - shorter treatment times (minutes versus days), and the protection of health care personnel from radiation exposure. Overall clinical outcomes and toxicities are felt to be similar with both HDR and LDR [17]. 
A third type of treatment, not commonly used in the United States, is known as Pulsed-Dose Rate (PDR) brachytherapy [17]. This hybrid form of treatment uses an HDR source and remote afterloader to mimic the radiobiologic effects of LDR treatment, which is accomplished by deploying the source for a brief period of time, hourly, over a prolonged treatment delivery period (2-3 days). PDR has potential radiobiologic advantages, but it requires prolonged patient immobilization and hospitalization. PDR remains in use at several key academic centers, but patients treated with this technology represent only a small proportion of all cervical cancer patients who are treated with brachytherapy [17].

\section{Endometrial Cancer}

Endometrial cancer (EC) is a leading cause of female cancer in developed countries. It is the fifth most common cancer worldwide in women. In 2015 it is estimated that 54,870 women were diagnosed with and 10,170 died of endometrial cancer [18]. The primary management of endometrial cancer is total abdominal hysterectomy and bilateral salpingo-oopherectomy (TAH-BSO). The role of pelvic and para-aortic lymph node dissection is controversial in the surgical management of endometrial cancer [18]. Adjuvant radiation therapy for endometrial cancer is also controversial but is routinely recommended based upon presence of adverse risk factors such as higher stage, increased depth of myometrial invasion (MMI), higher grade, presence of lymphovascular space invasion (LVSI), increasing age, increasing tumor size, histology, and lymph node positivity [18]. The role of vaginal brachytherapy (VBT) in the post-operative management of endometrial cancer continues to evolve.

In inoperable endometrial cancer, those patients with severe comorbidities or advanced age, radiation therapy remains the only curative option. In the past, LDR brachytherapy with radium-226 was used in combination with external beam radiotherapy (EBRT) [19, 20]. More recently, LDR brachytherapy has been replaced by HDR brachytherapy with iridium-192 after loading [21, 22].

The classification as medically inoperable can be medical comorbidities such as cardiovascular disorders, pulmonary disease, cerebrovascular accidents, venothromboembolic disease, renal disease, or other more rare conditions, including Marfan syndrome, hemophilia, other malignancies, or age. Increasingly, patients are deemed to be medically inoperable because of morbid obesity [23]. This designation may relate to the surgeons' experience in operating on such patients and may be a relative contraindication rather than a strict contraindication.

The use of HDR brachytherapy in the treatment of uterine $\mathrm{CC}$ and $\mathrm{EC}$ has been increasing worldwide. In developing countries like Brazil, advantages of HDR in comparison to LDR brachytherapy include: outpatient treatment, potential cost savings, shorter treatment time, elimination of radiation exposure, patient comfort, reduction of the need for general anesthesia, and less chance of applicator displacement; which makes this procedure an excellent treatment option. Despite the radiobiological disadvantages of HDR brachytherapy, the possibility of optimizing dose distribution seems to outweigh the disadvantages [24]. The variation of dwell time with the single stepping source permits an almost infinite variation on the effective source strength and source positions, allowing for greater control of the dose distribution and potentially less morbidity [22]. Although HDR brachytherapy has been used for more than 30 years, there is still no consensus about the optimum fractionation schedule in the treatment of $\mathrm{CC}[21,25]$ and there is a wide variation in its clinical practice, as found by the ABS survey of brachytherapy practice for carcinoma of the cervix in the U.S. The medium HDR brachytherapy dose used in 315 institutions was 29 and 30 Gy for early and advanced cancers, respectively. The median dose per fraction was $6 \mathrm{~Gy}$ for a median of 5 fractions $[21,26]$. Due to the wide variation of treatment schedule in the literature, the ABS formed a committee to issue guidelines specifically for the use of HDR brachytherapy for cervical carcinoma and published its recommendations [22]. On this article, the ABS has suggested tables for combining the EBRT with HDR brachytherapy for patients with early and advanced stages of disease; however, the authors advise that the schedules suggested have not been thoroughly tested clinically.

According to Pellizzon [27], vaginal recurrence of EC is a risk, particularly in patients who do not receive adjuvant radiotherapy. For low risk endometrial patients that recur, the vagina is the sole site of failure in $30-50 \%$ of patients, and the most common location of recurrence is at the vaginal apex, with a relative frequency of $4: 1$.

Strategies to combat the growing CC burden in LMIC must include context-specific consideration of radiotherapy delivery in limited resource environments. As Suneja et al. [26] have demonstrated, CC standards of care need to be adapted to accommodate the unique circumstances of individual countries in order to maximize efficacy [28]. In their recent publication, Suneja et al. [26] adapted the ABS guidelines to address brachytherapy delivery in minimal resource settings [28]. A significant challenge that many LMIC face is the lack of access to advanced imaging technology. Such is the case in Rwanda, where the majority of stage IB to III CC patients are unable to undergo staging CT scans, due to the small number of scanners, expense and lengthy turnaround time. Importantly, Suneja et al. [26] take note of this barrier, advocating that effective planning and treatment administration can be accomplished with such constraints. The paper includes extensive recommendations for how to approach aspects such as treatment fields, planning and applicator placement 
without this imaging, such as using bony landmarks, prescribing to points of interest and using fixed applicator configurations with a plan library or radioopaque applicators [29].

\section{Brachytherapy alone in limited resource settings}

In 1915, Kelly and Burnham [28] published a study of 213 cervical and vaginal cancer patients treated with surgery and brachytherapy, or with brachytherapy alone [28]. Of the 203 patients treated with brachytherapy alone, 4 were considered operable and 199 were considered inoperable. All 4 operable patients, and 53 of the inoperable patients were considered cured following treatment, with no evidence of disease on palpation or curettage. Follow up intervals ranged from 6 years to 6 months, with 29 patients without disease at 1 year. Moreover, 109 patients experienced improvement of symptom management and reduction in tumor burden [30]. Kelly and Burnham [28] concluded that this improvement alone, regardless of cure, justified this application of radiotherapy in cervical and vaginal cancer.

Hamberger et al. [31] reported results from a study of $151 \mathrm{CC}$ patients treated with brachytherapy alone. 5 year overall survival rates of 100,96 and $86 \%$ were achieved for patients with stage IA, IB small volume and IB disease respectively [32]. Regional recurrences occurred in 7 patients, while only one distant metastasis was observed. Hamberger et al. [31] proposed that brachytherapy alone was capable of covering an anatomical area equivalent to radical hysterectomy, and that the addition of EBRT would only increase the irradiated region and subsequent risk of fibrosis [32]. They argued that given the low rates of observed failure, brachytherapy alone was justified in Stage I disease.

According to the American Society of Clinical Oncology's 2016 resource stratified clinical practice guidelines, areas with brachytherapy but no EBRT should be classified as equivalent to a "Basic" level of radiotherapy, defined as unavailable radiation treatment [33]. Machine wear and tear, financial constraints and infrastructural capacity are all potent drivers of such a scenario and are not infrequent in LMIC. Brachytherapy alone, with low dose cis-platinum chemotherapy followed by radical hysterectomy, remains as only low evidence, weak recommendation for IB2 and IIA2 disease [33].

The implementation of brachytherapy is not without limitations. The technology requires significant safety and maintenance considerations. As decaying radioactive sources, brachytherapy units must not only be stored appropriately, but also replaced over time. Operators must therefore be extensively trained in both operation and upkeep of units in order to ensure treatment efficacy and safety. Nandwana et al. [30] demonstrated the ability of cobalt- 60 to meet the necessary qualifications as an intracavitary radiotherapy source under the International Commision on Radiation Units guidelines [30]. Thus, cobalt-60 could be considered as an alternative to iridium-192 in limited resource settings in order to decrease the frequency of source exchange and potential customs complications.

\section{CC treatment in the developing world}

The majority of patients with $\mathrm{CC}$ in the developing world present with an advanced stage of disease, with limited access to adequate treatment resulting in high mortality rates for these women.

Fractionated HDR brachytherapy has a potential to reduce the incidence of late complications caused by high doses delivered by intracavitary brachytherapy to rectum and bladder. Despite its radiobiological disadvantages mentioned by Eifel [24], the possibility of optimizing dose distribution and the lesser chance of applicator displacement seem to outweigh these disadvantages. Furthermore, the variation of dwell time with the single stepping source permits an almost infinite variation on the effective source strength and source positions, which allows for greater control of dose distribution and potentially less morbidity [18].

No data in the literature show a higher incidence of late complications in patients with CC treated with HDR brachytherapy compared with those treated with LDR. The incidences of lower 5-year rectal complications in patients from the HDR group were probably the result of the relatively low dose delivered to the rectum with the HDR brachytherapy fractionation. In LDR brachytherapy, the total rectal dose is commonly limited to 70 Gy [18]. The optimization of HDR brachytherapy can be further improved with 3D CT or MRI imaging to increase the delivered dose to the target volume, while minimizing the dose to the adjacent normal tissues [18].

\section{Conclusion}

Brachytherapy is considered a safe technique for patients with cervical and endometrial cancer and with very satisfactory results of regression and even tumor healing and with less toxicity to adjacent normal tissues.

\section{Abbreviations \\ ABS: American Brachytherapy Society; BSO: Bilateral Salpingo - Oophorectomy; CC: Cervical Cancer; CT: Computer Tomography; EBRT: External Bean Radiotherapy; EC: Endometrial Cancer; Gy: Gay; HDR: High Dose Rate; LDR: Low Dose Rate; LMIC: Low and middle income countries; LVSI: Lynphovascular Space; MMI: Myometrial Invasion; MRI: Magnetic Resonance Imaging; NCCN: National Comprehensive Cancer Network; PDR: Pulsed Dose Rate; TAH: Total Abdominal Hysterectomy; US: Ultrasonography; VBT: Vaginal Brachytherapy}

\section{Acknowledgements}

Antonio Cassio Assis Pellizzon and Fabian Friedrich by correction of the manuscript. 


\section{Funding}

There was no funding provided for this study.

\section{Availability of data and materials}

The data that support the findings of this study are available from https:// www.ncbi.nlm.nih.gov/pubmed.

\section{Authors' contributions}

$M U, M D$ was responsible for the research and writing of this manuscript. FF $\mathrm{MD}, \mathrm{PhD}$ was responsible for the research and revision of this manuscript. ACAP, MD; PhD was responsible for the revision of this manuscript. All authors read and approved the final manuscript.

\section{Ethics approval and consent to participate}

\section{Not Applicable.}

\section{Consent for publication}

I, Mari Uyeda give my consente for publishing the literature review entitled: High Dose Rate (HDR) brachytherapy in the Gynecological Cancer: a literature review.

I, Fabian Friedrich give my consente for publishing the literature review entitled: High Dose Rate (HDR) brachytherapy in the Gynecological Cancer: a literature review

I, Antonio Cassio Assis Pellizzon, MD; PhD give my consente for publishing the literature review entitled: High Dose Rate (HDR) brachytherapy in the Gynecological Cancer: a literature review.

\section{Competing interests}

The authors declare that they have no competing interests.

\section{Publisher's Note}

Springer Nature remains neutral with regard to jurisdictional claims in published maps and institutional affiliations.

\section{Author details}

${ }^{1}$ AC Camargo Cancer Center, Tamandaré St, 753 - Liberdade, São Paulo 01509-020, Brazil. ²Department of Radiotherapy of the Hospital AC Camargo Cancer Center, São Paulo, Brazil. ${ }^{3}$ Health of the Municipality of Blumenau Dois de Setembro St, Itoupava Norte, Blumenau, SC 89052-001, Brazil. ${ }^{4}$ Health of the Municipality of Blumenau, Blumenau, Santa Catarina, Brazil.

\section{Received: 11 September 2018 Accepted: 2 December 2018} Published online: 14 December 2018

\section{References}

1. Esteves SCB, Oliveira ACZ, Feijó LFA. Braquiterapia de Alta Taxa de Dose no Brasil. Radiol Bras. 2004;37(5):337-41.

2. Bailey HH, Chuang LT, DuPont NC, Eng C, Foxhall LE, Merrill JK, Wollins DS, Blanke CD. American society of clinical oncology statement: human papillomavirus vaccination for cancer prevention. J Clin Oncol. 2016;34: 1803-12.

3. Tewari KS, Monk BJ. Invasive cervical cancer. In: DiSaia PJ, Creasman WT, editors. Clinical gynecologic oncology. 8th ed. Philadelphia: Mosby; 2012

4. Monk BJ, Tewari KS, Koh WJ. Multimodaity therapy for locally advanced cervical carcinoma: state of the art and future directions. J Clin Oncol. 2017;25:2952-65.

5. Bosch FX, Broker TR, Forman D. Comprehensive control of human papillomavirus infections and related diseases. Vaccine. 2013;31:11-31.

6. Sadalla JC, Andrade JM, Gente MLND, Baracat EC. Cervical cancer: what's new? Rev Assoc Med Bras. 2015;61(6):536-42.

7. Kato T, Takashima A, Kasamatsu T, Nakamura K, Mizusawa J, Nakanishi T, et al. Clinical tumor diameter and prognosis of patients with FIGO stage IB1 cervical cancer (JCOG0806-a). Gynecol Oncol. 2015;137(1):34-9.

8. Halaska MJ, Robova H, Pluta M, Rob L. The role of trachelectomy in cervical cancer. E Cancer Medical Science. 2015:9:506.

9. Small W Jr, Bacon MA, Bajaj A, Chuang LT, Fisher BJ, Harkenrider MM, Jhingran A, Kitchener HC, Mileshkin LR, Viswanathan AN, Gaffney DK. Cervical Cancer: A Global Health Crisis. Cancer. 2017;123(13):2404-12.

10. Querleu D, Morrow CP. Classification of radical hysterectomy. Lancet Oncol. 2008;9(3):297-303.
11. Petsuksiri J, Chansilpa Y, Therasakvichya S, Suntornpong N, Thephamongkhol K, Dankulchai $\mathrm{P}$, et al. Treatment options in bulky stage IB cervical carcinoma. Int J Gynecol Cancer. 2008;18(6):1153-62.

12. Minig L, Patrono MG, Romero N, Rodríguez Moreno JF, Garcia-Donas J. Different strategies of treatment for uterine cervical carcinoma stage IB2- IIB. World J Clin Oncol. 2014;5(2):86-92.

13. LaVignea AW, Triedmanb SA, Randallc TC, Trimbled EL, Viswanathan AN Cervical cancer in low and middle income countries: addressing barriers to radiotherapy delivery. Gynecologic Oncology Reports. 2017;22:16-20.

14. IAEA. Inequity in Cancer Care: A Global Perspective. 2011.

15. Cetina L, González-Enciso A, Cantú D, Coronel J, Pérez-Montiel D, Hinojosa J, Serrano A, Rivera L, Poitevin A, Mota A, Trejo E, Montalvo G, Muñoz D, RoblesFlores J, De La Garza J, Chanona J, Jiménez-Lima R, Wegman T, DueñasGonzález A. Brachytherapy versus radical hysterectomy after external beam chemoradiation with gemcitabine plus cisplatin: a randomized, phase III study in IB2-IIB cervical cancer patients. Ann Oncol. 2013;24:2043-7.

16. Zubizarreta $\mathrm{EH}$, Fidarova $\mathrm{E}$, Healy $\mathrm{B}$, Rosenblatt $\mathrm{E}$. Need for radiotherapy in low and middle income countries - the silent crisis continues. Clin Oncol. 2015;27:107-14.

17. Banerjee R, Kamrava M. Brachytherapy in the treatment of cervical cancer: a review. Int J Women's Health. 2014;6:555-64.

18. Sabater S, Andres I, Honrubia VL, Berenguer R, Sevillano M, Jimenez EJ, Rovirosa A, Arenas M. Vaginal cuff brachytherapy in endometrial cancer - a technically easy treatment? Cancer Manag Res. 2017;9:351-62.

19. Chao CK, Grigsby PW, Perez CA, Camel HM, Kao MS, Galakatos AE, et al. Brachytherapy-related complications for medically inoperable stage I endometrial carcinoma. Int J Radiat Oncol Biol Phys. 1995:31:37-42.

20. Kucera H, Sagl R, Skodler W, Weghaupt K. Afterloading short-term irradiation of inoperable uterine cancer. Geburtshilfe Frauenheilkd. 1986;46:515-9.

21. Nag S, Erickson B, Thomadsen B, et al. The American brachytherapy society recommendations for high-dose-rate brachytherapy for carcinoma of the cervix. Int J Radiat Oncol Biol Phys. 2000;48:201-21.

22. Schwarz JK, Beriwal S, Esthappan J, Erickson B, Feltmate C, Fyles A, Gaffney D, Jones E, Klopp A, Small W Jr, Thomadsen B, Yashar C, Viswanathan A. Consensus statement for brachytherapy for the treatment of medically inoperable endometrial cancer. Brachytherapy. 2015;14:587e599.

23. Petereit DG, Pearcey R. Literature analysis of high dose rate brachytherapy fractionation schedules in the treatment of cervical cancer: is there an optimal fractionation schedule? Int J Radiat Oncol Biol Phys. 1999:43:359-66.

24. Eifel PJ. High-dose-rate brachytherapy for carcinoma of the cervix: high tech or high risk? Int J Radiat Oncol Biol Phys. 1992;24:383-6.

25. Ferrigno R, Novaes PERS, Pellizzon ACA, Maia MAC, Fogarolli RC, Gentil AC, Salvajoli JV. High-Dose-Rate Brachytherapy In The Treatment Of Uterine Cervix Cancer. Analysis Of Dose Effectiveness And Late Complications. Int. J. Radiation Oncology Biol. Phys. 2000;50:1123-35.

26. Suneja G, Brown D, Chang A, Erickson B, Fidarova E, Grover S, Mahantshetty U, Nag S, Narayan K, Bvochora-Nsingo M, Viegas C, Viswanathan AN, Lin MY, Gaffney D. American brachytherapy society: brachytherapy treatment recommendations for locally advanced cervix cancer for low-income and middle-income countries. Brachytherapy. 2017:16:85-94.

27. Pellizzon ACA, Miziara M, Fogaroli R, Soares C, Baraldi H. Vault high dose rate brachytherapy for low to intermediate risk endometrial adenocarcinoma completely resected. Int J Cancer (Radiat Oncol Invest). 2002;96(Suppl):105-8.

28. Kelly HA, Burnam CF. Radium in the treatment of carcinomas of the cervix uteri and vagina. JAMA. 1915;15:1874-8.

29. Chuang LT, Temin S, Camacho R, Feldman S, Gultekin M, Gupta V, Horton S, Jacob G, Kidd EA, Lishimpi K, Nakisige C, Nam J-H, Sheung NY, Small W, Thomas G, Berek JS. Management and care of women with invasive cervical cancer: American Society of Clinical Oncology resource-stratified clinical practice guideline. J Global Oncol. 2016;2:311-40.

30. Nandwana U, Rathore N, Gupta S, Shukla A, Kumar S, Intodia K, Chand Bana $P$, Jain A. Cobalt-60 is a logical, economical and comparable alternative to Ir- 192: analysis and institutional experience from western India. South African J Gynaecol Oncol. 2015;7:60-3.

31. Hamberger A, Fletcher G, Wharton J. Results of treatment of early stage I carcinoma of the uterine cervix with intracavitary radium alone. Cancer. 1978;41:980-5.

32. Koh WJ, Greer BE, Abu-Rustum NR, et al. Cervical cancer, version 2.2015. J Natl Compr Canc Netw. 2015;13:395-404.

33. Ferrigno R, Nishimoto IN, Novaes PERS, Pellizzon ACA, Maia MAC, Fogarolli RC, Salvajoli JV. Comparison Of Low And High Dose Rate Brachytherapy In The Treatment Of Uterine Cervix Cancer. Int. J. Radiation Oncology Biol. Phys. 2005;62:1108-16. 